\title{
Multiply stochastic representations for $K$ distributions and their Poisson transforms
}

\author{
Malvin C. Teich and Paul Diament \\ Department of Electrical Engineering, Columbia University, New York, New York 10027
}

Received March 10, 1988; accepted July 25, 1988

\begin{abstract}
The $K$ distribution is used in a number of areas of scientific endeavor. In optics, it provides a useful statistical description for fluctuations of the irradiance (and the electric field) of light that has been scattered or transmitted through random media (e.g., the turbulent atmosphere). The Poisson transform of the $K$ distribution describes the photon-counting statistics of light whose irradiance is $K$ distributed. The $K$-distribution family can be represented in a multiply stochastic (compound) form whereby the mean of a gamma distribution is itself stochastic and is described by a member of the gamma family of distributions. Similarly, the family of Poisson transforms of the $K$ distributions can be represented as a family of negative-binomial transforms of the gamma distributions or as Whittaker distributions. The $K$ distributions have heretofore had their origins in random-walk models; the multiply stochastic representations provide an alternative interpretation of the genesis of these distributions and their Poisson transforms. By multiple compounding, we have developed a new transform pair as a possibly useful addition to the $K$-distribution family. All these distributions decay slowly and are difficult to calculate accurately by conventional formulas. A recursion relation, together with a generalized method of steepest descent, has been developed to evaluate numerically the photon-counting distributions and their factorial moments with excellent accuracy.
\end{abstract}

\section{INTRODUCTION}

As with so many of the probability distributions of current interest in optics and information transmission, the $K$ distribution was introduced first in entomology. In 1953, Broadbent and Kendall ${ }^{1}$ used the $K_{0}$ distribution to describe the spatial distribution of certain larvae in terms of a two-dimensional random-walk model coupled with an exponentially distributed stopping time. ${ }^{2}$ In 1975, Yasuda $^{3}$ generalized the result of Broadbent and Kendall by using a gammadistributed stopping time in the Rayleigh random walk, obtaining the $K$ distribution. He then used this to describe the behavior of matrimonial distances (the distance between the birthplaces of mates) in Japan and Italy. The appellation " $K$ distribution" appears to have been used first by Malécot ${ }^{4}$ in 1967.

In optics, the $K_{0}$ distribution was used first in 1970 by Bertolotti et al..$^{5}$ in connection with the problem of doubly scattered laser light. A more general approach, initiated by Pusey, Jakeman, and their colleagues in 1976, made use of the $K$ distribution for problems involving the field and irradiance in scattering, speckle, and the propagation of light through turbulent media. ${ }^{6-12}$ The same model was used also to describe non-Rayleigh microwave sea echo. ${ }^{13-15}$ A number of generalizations of the $K$ distribution, including the $I-$ $K$ distribution ${ }^{16,17}$ and the generalized $K$ distribution, ${ }^{18,19}$ were set forth recently. The $K$ distribution also was obtained in the context of a quantum-mechanical formulation. ${ }^{20}$ The smoothing of the $K$ distribution caused by dead time $^{21}$ and saturation ${ }^{22}$ has been considered as well.

Two physically distinct doubly stochastic representations were developed for the $K$ distribution and for some of its generalizations. In the first, considered by Yasuda ${ }^{3}$ and pursued by a number of researchers, ${ }^{12,13,17,19,23-26}$ the second moment of a Rayleigh distribution is smeared by a gamma distribution. In the second, considered by Lewinski, ${ }^{14}$ the mean of a gamma distribution is smeared by another gamma distribution. It was shown by Jakeman and Tough ${ }^{19}$ that the generalized $K$ distribution can be represented as a Rician distribution with both its mean-squared noise component and its coherent amplitude varying in correlated fashion, according to a gamma distribution.

The Poisson transform of the $K$ distribution, describing the photon-counting detection of light whose field (or irradiance) is $K$ distributed, has been written in terms of Whittaker functions. $5,6,21,27-29$

The purpose of this paper is twofold. First, we show that the family of $K$ distributions and their Poisson transforms can be represented as multiply stochastic distributions, in which the mean of one distribution is smeared by another. For the $K^{\prime}$ distribution, which is a generalization of the $K$ distribution, the mean of a gamma distribution is smeared by another gamma distribution; for the Poisson transform of the $K^{\prime}$ distribution, the mean of a negative-binomial distribution is smeared by a gamma distribution. Second, we obtain mathematical expressions and numerical results for the Poisson transforms of the $K$ family (i.e., for the photoncounting distributions of light whose irradiance fluctuations are $K$ distributed).

A schematic representation for the process is provided in Fig. 1(a). A scattering (or atmospheric) medium of fluctuating transmission (with characteristic fluctuation time $\tau_{a}$ ) imposes a random modulation on the mean irradiance $W$ of a light source that passes through it. For purposes of illustration, we take the fluctuations of the mean irradiance imposed by the medium to be gamma distributed. These fluctuations result in an overall mean $a W$ for the light that emerges from the medium, where $a$ is a scaling factor introduced by the medium. Light sources also exhibit intrinsic irradiance fluctuations (characterized by the coherence time 


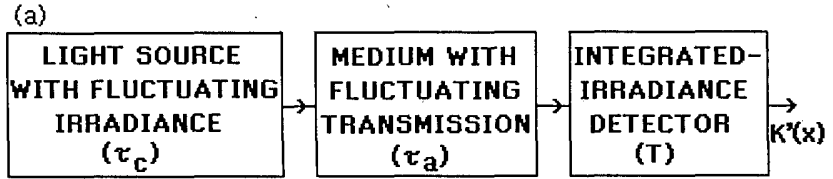

(b)

\begin{tabular}{|c|c|c|}
\hline & & \\
\hline $\begin{array}{l}\text { LIGHT SOURCE } \\
\text { WITH FLUCTUATING }\end{array}$ & $\begin{array}{l}\text { MEDIUM WITH } \\
\text { FLUCTUATING }\end{array}$ & $\begin{array}{l}\text { PHOTON- } \\
\text { COUNTING }\end{array}$ \\
\hline $\begin{array}{c}\text { IRAADIANCE } \\
\left(\tau_{C}\right)\end{array}$ & $\begin{array}{c}\text { TRANSMISSION } \\
\left(\tau_{a}\right)\end{array}$ & $\begin{array}{l}\text { DETECTOR } \\
\text { (T) }\end{array}$ \\
\hline
\end{tabular}

Fig. 1. Models representing the effects of a medium with fluctuating transmission (or a scattering medium) on the statistical properties of a light source observed at the output of a detector. (a) The medium (with characteristic fluctuation time $\tau_{a}$ ) stochastically modulates the mean irradiance of the source, the source exhibits intrinsic irradiance fluctuations (with coherence time $\tau_{c}$ ), and the detector records the overall (continuous) integrated irradiance (in the time $T$ ). (b) Same as (a) but now the detector records the overall (discrete) photon count (in the time $T$ ).

$\tau_{c}$ ). The integrated irradiance of chaotic (or thermal) light, for example, is gamma distributed. A gamma-fluctuating light source, whose mean is smeared by a random medium in accordance with another gamma distribution, results in a doubly stochastic distribution for the integrated intensity that is precisely the $K^{\prime}$ distribution. A unity-quantumefficiency detector of the integrated irradiance provides a direct measure of this distribution. Equivalently, the gamma-distributed irradiance fluctuations may be viewed as modulating the mean of the gamma-distributed transmission of the random medium.

In the scheme shown in Fig. 1(b), the fluctuating medium and the light source have the same properties as those considered above. The integrated intensity is therefore again describable by the $K^{\prime}$ distribution, but in this case a photoncounting detector is used. It measures the Poisson transform of the integrated intensity rather than the integrated intensity itself. The resultant discrete photon-counting distribution, which we refer to as $A(n)$, is therefore the Poisson transform of the $K^{\prime}$ distribution. For a unity-quantumefficiency counter, it registers the same overall mean as the integrated-irradiance detector.

After discussing the general properties of multiply stochastic distributions in Section 2, we proceed to develop doubly stochastic representations for the $K$-distribution family in Section 3 and to obtain Poisson transforms of these representations in Section 4. In Sections 5 and 6 we calculate and present the photon-counting distributions $A(n)$. The conclusion is provided in Section 7.

\section{MULTIPLY STOCHASTIC DISTRIBUTIONS}

A multiply stochastic distribution is formed from a simple one by smearing (averaging) its mean over a first-level distribution; the latter may itself have a mean that is subject to uncertainty and is then averaged over a second-level distribution of that mean. The process may need to be continued, in principle, until the deepest-level distribution has a mean and other statistical parameters that are truly deterministic. A vastly expanded variety of random behavior can thereby be accounted for and related to the underlying probabilistic nature of the processes involved. Multiply stochastic distributions have been applied in a variety of disciplines. ${ }^{27,30}$

The complexity of the probability distributions tends to rise rapidly with each additional level of smearing of the mean, and the mathematical expressions for the resultant counting distributions soon become difficult to calculate and intractable to analysis. Parameters other than the mean of the distributions may be smeared, giving rise to other families of distributions.

\section{A. Notational Conventions}

To simplify the notation and the manipulations of the expressions required for the calculations, we adhere to the following conventions.

A probability distribution at any level is written as an appropriately selected symbol for the function, with the independent random variable as its first argument and its mean as its last argument; other parameters of the distribution appear between the first and last ones. For example, the gamma distribution for a continuous variable $x$ with mean $N$ and degrees-of-freedom parameter $\alpha$ is designated $G(x, \alpha, N)$, as an abbreviation for its full mathematical form, given by

$$
G(x, \alpha, N)=\left[(\alpha / N)^{\alpha} / \Gamma(\alpha)\right] x^{\alpha-1} \exp (-\alpha x / N) .
$$

It is understood implicitly that the range of $x$ is 0 to $\infty$ and that $\alpha>0$ to ensure that its integrals converge.

In the notation introduced by Gurland, ${ }^{31}$ the process of smearing the mean of one distribution by averaging over another is abbreviated by the operator $\wedge$, defined as an integration (or summation in the discrete case) over the adjacent repeated parameter. This is akin to the Einstein convention for repeated indices in tensor analysis. The results are referred to sometimes as compound or mixed distributions, but we prefer the term doubly stochastic distributions.

\section{B. Example}

An example is provided by the doubly stochastic counting distribution that results when a Poisson counting process with mean $W$, which has the distribution $P(n, W)=\left(W^{n} /\right.$ $n !) e^{-W}$, is smeared by allowing its mean $W$ to take on values associated with a gamma distribution. This is expressed by

$$
p(n, \alpha, N)=P(n, W) \wedge G(W, \alpha, N),
$$

which, because $W$ is the repeated symbol that becomes the dummy variable of integration, stands for the averaging operation

$$
\begin{aligned}
p(n, \alpha, N)= & \int_{0}^{\infty} P(n, W) G(W, \alpha, N) \mathrm{d} W \\
= & \int_{0}^{\infty}\left(W^{n} / n !\right) e^{-W}\left[(\alpha / N)^{\alpha} / \Gamma(\alpha)\right] W^{\alpha-1} \\
& \times \exp (-\alpha W / N) \mathrm{d} W .
\end{aligned}
$$

In this instance, the integration presents no difficulties and results in the negative-binomial distribution

$$
\begin{aligned}
p(n, \alpha, N) & =B(n, \alpha, N) \\
& =\left[\alpha^{\alpha} / \Gamma(\alpha)\right][\Gamma(n+\alpha) / n !] N^{m} /(N+\alpha)^{n+\alpha} .
\end{aligned}
$$


The notation $p(n, \alpha, N)=P(n, W) \wedge G(W, \alpha, N)$ is consistent with the first convention, as $N$ remains the mean of the resultant distribution $p(n, \alpha, N)$, necessarily, since $W$ is the mean of $P(n, W)$ and $N$ is the mean of $G(W, \alpha, N)$ :

$$
\begin{aligned}
\langle n\rangle & =\sum n p(n, \alpha, N)=\sum n \int P(n, W) G(W, \alpha, N) \mathrm{d} W \\
& =\int \sum n P(n, W) G(W, \alpha, N) \mathrm{d} W \\
& =\int W G(W, \alpha, N) \mathrm{d} W=N
\end{aligned}
$$

The summations over $n$ and the integrations over $W$ both span the entire range from 0 to $\infty$.

When we wish to allow for a gain (or loss) factor, $a$, in the overall mean, we can maintain the notational convention with this gain factor included, as in

$$
p(n, \alpha, a N)=P(n, a W) \wedge G(W, \alpha, N),
$$

where the integration is again over the repeated variable $W$. This is found to be the negative-binomial distribution with modified mean, $B(n, \alpha, a N)$. More generally, if the means of two compounded distributions undergo linear transformations, then so does the mean of the resultant one, as in

$$
h\left(x, a_{3} N+b_{3}\right)=f\left(x, a_{1} u+b_{1}\right) \wedge g\left(u, a_{2} N+b_{2}\right),
$$

with

$$
a_{3}=a_{1} a_{2}, \quad b_{3}=a_{1} b_{2}+b_{1}
$$

\section{Moments}

The abbreviated notation becomes even more powerful when the $\wedge$ operation is extended to include expressions that are not themselves probability distributions but are involved in the calculations of means, variances, and moments of the distributions. Thus the above calculation of the mean of the distribution can consistently be abbreviated as

$$
\begin{aligned}
\langle n\rangle & =n \wedge p(n, \alpha, N)=n \wedge P(n, W) \wedge G(W, \alpha, N) \\
& =W \wedge G(W, \alpha, N)=N .
\end{aligned}
$$

Similarly, the calculation of moments of continuous distributions, or of factorial moments for discrete ones, can be abbreviated as

$$
\left\langle x^{n}\right\rangle=x^{n} \wedge p(x, N)=\int x^{n} p(x, N) \mathrm{d} x,
$$

and, defining $f(m, n)$ as the factorial ratios, i.e.,

$$
f(m, n)=n ! /(n-m) !=n(n-1)(n-2) \cdots(n-m+1) \text {, }
$$

we obtain the factorial moments for a discrete distribution in the form

$$
\langle f(m, n)\rangle=\langle n ! /(n-m) !\rangle=f(m, n) \wedge p(n, N) .
$$

For example, for the Poisson distribution, the factorial moments are

$$
f(m, n) \wedge P(n, N)=N^{m} .
$$

It follows at once that if a Poisson distribution is compounded with any continuous distribution $p(x, M)$ to form the discrete one $F(n, M)=P(n, x) \wedge p(x, M)$, then the factorial moments of $F$ are identical to the direct moments of $p$ :

$$
\begin{aligned}
f(m, n) \wedge F(n, M) & =f(m, n) \wedge P(n, x) \wedge p(x, M) \\
& =x^{m} \wedge p(x, M)=\left\langle x^{m}\right\rangle .
\end{aligned}
$$

This corresponds to a well-known theorem relating the two sets of moments.

\section{Symmetry}

The compounding operation is not only associative but also generally symmetric, or commutative, in the sense that

$$
h_{1}(x, N)=f(x, u) \wedge g(u, N)
$$

is the same distribution as

$$
h_{2}(x, N)=g(x, u) \wedge f(u, N) .
$$

This property will hold if the constituent distributions $f(x$, $N)$ and $g(x, N)$ are such that their moments $\left\langle x^{m}\right\rangle$ are proportional to $N^{m}$, as is borne out by a dimensional analysis, based on $\langle x\rangle=N$. If this is so, i.e., if

$$
\left\langle x^{m}\right\rangle=F_{m} N^{m} \quad \text { for } f(x, N)
$$

and

$$
\left\langle x^{m}\right\rangle=G_{m} N^{m} \quad \text { for } g(x, N)
$$

then the moments of the two versions of $h(x, N)$ are, for $h_{1}(x$, $N)=f \wedge g$,

$$
\left\langle x^{m}\right\rangle=F_{m} u^{m} \wedge g(u, N)=F_{m}\left\langle u^{m}\right\rangle=F_{m} G_{m} N^{m}
$$

and, for $h_{2}(x, N)=g \wedge f$,

$$
\left\langle x^{m}\right\rangle=G_{m} u^{m} \wedge f(u, N)=G_{m}\left\langle u^{m}\right\rangle=G_{m} F_{m} N^{m} .
$$

Since all the moments of the two version of the $h(x, N)$ distribution are the same, we conclude that $h_{1}=h_{2}$ and the compounding operation is commutative.

\section{DOUBLY STOCHASTIC REPRESENTATIONS FOR THE IRRADIANCE DISTRIBUTIONS}

A number of continuous probability-density functions (pdf's) of interest in our study, and their direct moments, are presented in Table 1. Various equivalent representations for the pdf's are also shown. The entries under the heading Equivalent Density Functions explain the provenance of the distributions, as a smearing of the mean of a more primitive distribution with another one. We have used the standard notations ${ }^{32}$ for the gamma function $\Gamma(x)$, the generalized Laguerre polynomials $L_{n}{ }^{(\alpha)}(x)$, and the modified Bessel functions of the first kind $I_{n}(x)$ and of the second $\operatorname{kind} K_{n}(x)$. Several well-known entries ${ }^{27}$ are included in the table, to facilitate comparisons.

The distribution denoted by $g(x, \alpha)$ and labeled the basic gamma is the gamma distribution stripped of the $\alpha / N$ factor that distinguishes its mean from its parameter; that is, the gamma distribution $G(x, \alpha, N)$ is merely a rescaling of the basic gamma distribution: $G(x, \alpha, N)=(\alpha / N) g(\alpha x / N, \alpha)$. 
Table 1. Pdf's, Moments, and Equivalent Density Functions for Some Continuous Random Variables

\begin{tabular}{|c|c|c|c|c|}
\hline Name & Symbol & Expression (pdf) & Moments $\left\langle x^{m}\right\rangle$ & $\begin{array}{c}\text { Equivalent } \\
\text { Density Functions }\end{array}$ \\
\hline Delta & $D(x, N)$ & $\delta(x-N)$ & $N^{m}$ & $G(x, \infty, N)$ \\
\hline Exponential & $E(x, N)$ & $(1 / N) \exp (-x / N)$ & $N^{m} m !$ & $G(x, 1, N)$ \\
\hline Gamma & $G(x, \alpha, N)$ & {$[(\alpha / N) / \Gamma(\alpha)](\alpha x / N)^{\alpha-1} \exp (-\alpha x / N)$} & $N^{m}\left[\Gamma(m+\alpha) / \alpha^{m} \Gamma(\alpha)\right]$ & $(\alpha / N) g(\alpha x / N, \alpha)$ \\
\hline Basic gamma & $g(x, \alpha)$ & {$[1 / \Gamma(\alpha)] x^{\alpha-1} e^{-x}$} & $\Gamma(m+\alpha) / \Gamma(\alpha)$ & $G(x, \alpha, \alpha)$ \\
\hline $\begin{array}{l}\text { Noncentral } \\
\text { chi-square }\end{array}$ & $I(x, \alpha, u+\alpha)$ & $(x / u)^{(\alpha-1) / 2} \exp [-(x+u)] I_{\alpha-1}\left[2(x u)^{1 / 2}\right]$ & $m ! L_{m}^{(\alpha-1)}(-u)$ & $g(x, k+\alpha) \wedge P(k, u)$ \\
\hline$K_{0}$ distribution & $K(x, N)$ & $(2 / N) K_{0}\left(2(x / N)^{1 / 2}\right)$ & $N^{m}(m !)^{2}$ & $K(x, 1, N) ; \quad E(x, u) \wedge E(u, N)$ \\
\hline$K$ distribution & $K(x, \alpha, N)$ & $\begin{array}{l}{[2(\alpha / N) / \Gamma(\alpha)](\alpha x / N)^{(\alpha-1) / 2}} \\
\quad \times K_{\alpha-1}\left(2(\alpha x / N)^{1 / 2}\right)\end{array}$ & $\begin{array}{l}N^{m} m ![\Gamma(m+\alpha) / \\
\left.\quad \alpha^{m} \Gamma(\alpha)\right]\end{array}$ & $E(x, u) \wedge G(u, \alpha, N)$ \\
\hline$K^{\prime}$ distribution & $K^{\prime}(x, \beta, \alpha, N)$ & $\begin{array}{l}{[2(\alpha \beta / N) / \Gamma(\alpha) \Gamma(\beta)](\alpha \beta x / N)^{[(\alpha+\beta) / 2]-1}} \\
\quad \times K_{\alpha-\beta}\left(2(\alpha \beta x / N)^{1 / 2}\right)\end{array}$ & $\begin{array}{l}N^{m}\left[\Gamma(m+\beta) / \beta^{m} \Gamma(\beta)\right] \\
\quad \times\left[\Gamma(m+\alpha) / \alpha^{m} \Gamma(\alpha)\right]\end{array}$ & $G(x, \beta, u) \wedge G(u, \alpha, N)$ \\
\hline GI distribution & $J(x, \beta, \alpha, u+\alpha)$ & $G(x, \beta, v) \wedge g(v, k+\alpha) \wedge P(k, u)$ & $\begin{array}{r}{\left[\Gamma(m+\beta) / \beta^{m} \Gamma(\beta)\right]} \\
\quad \times m ! L_{m}^{(\alpha-1)}(-u)\end{array}$ & $G(x, \beta, v) \wedge I(v, \alpha, u+\alpha)$ \\
\hline
\end{tabular}

The basic gamma distribution can be considered a continuous version of the discrete Poisson distribution, in that it reduces to the Poisson distribution when $\alpha$ is restricted to be an integer:

$$
g(x, n+1)=P(n, x) .
$$

The distribution $I(x, \alpha, u+\alpha)$ is the noncentral $\chi^{2}$ distribution (also called the generalized Rician-square distribution or the Laha distribution ${ }^{33}$ ). It applies to an optical source that emits a superposition of interfering coherent and chaotic radiation, with $\alpha$ (often denoted by $M$ ) as the number of degrees of freedom (or modes) and $u=\alpha I_{\text {coh }} / I_{\text {ch }}$, the number of modes times the ratio of coherent irradiance to chaotic irradiance. ${ }^{34}$ In this case, the equivalent doubly stochastic distribution $g(x, k+\alpha) \wedge P(k, u)$ involves a summation over the discrete index $k$, not an integration; the noncentral $\chi^{2}$ distribution is a compounding of the basic gamma distribution with a Poisson distribution. ${ }^{35}$ The notation used adheres to the convention that the last parameter, here $u+\alpha$, be the mean of the distribution.

The three $K$ distributions listed are denoted by $K_{0}, K$, and ' $K$ '. The first is the simplest, resulting from an exponential distribution that smears another exponential, $E(x, u) \wedge E(u$, $N)$. The second is the $K$ distribution that is usually cited, which is obtained from an exponential distribution smeared by (or driven by) a gamma distribution. This was considered initially to arise from a Rayleigh distribution,

$$
R(x)=(2 / S) x \exp \left(-x^{2} / S\right)
$$

whose second moment $S=\left\langle x^{2}\right\rangle$ is randomized by a gamma distribution. ${ }^{3,12,13,17,19,23-26}$ The equivalence follows from the fact that the square of a variable with a Rayleigh distribution exhibits an exponential distribution. The more general $K^{\prime}$ distribution results from a gamma distribution smeared by another gamma distribution. The parameters $\alpha$ and $\beta$ may be viewed as degrees-of-freedom parameters, ${ }^{27}$ such that

$$
\alpha \approx 1 \quad \text { if } T \ll \tau_{c} \quad \text { and } \quad \alpha \approx T / \tau_{c} \quad \text { if } T \gg \tau_{c},
$$

$$
\beta \approx 1 \quad \text { if } T \ll \tau_{a} \quad \text { and } \quad \beta \approx T / \tau_{a} \quad \text { if } T \gg \tau_{a} .
$$

As indicated above, the $K^{\prime}$ distribution, $G(x, \beta, u) \wedge G(u, \alpha$, $N$ ), is symmetric in the two degrees-of-freedom parameters, $\alpha$ and $\beta$.

The distribution denoted $G I$ in Table 1, a compounding of the gamma and the noncentral $\chi^{2}$ distributions, is also a compounding of two gamma distributions with a Poisson distribution. This can model the smearing of laser light that has a coherent component and an interfering chaotic component. When the coherent portion is eliminated $(u=0)$, this distribution reduces properly to $G(x, \beta, v) \wedge g(v, \alpha)$, which, except for a linear transformation of the mean, corresponds to the $K^{\prime}$ distribution.

The generalized $K$ distribution, derived from an $n$-dimensional random-walk model of weak scattering, ${ }^{18,19}$ can be expressed in terms of a smearing operation that involves the $K^{\prime}$ distribution. The generalized $K$ distribution can be written as

$$
\begin{aligned}
K_{G}(x, \alpha, \beta, \alpha \beta+\alpha(\alpha+1) z)= & {[2 / \Gamma(\alpha)] f_{\beta-1}(\sqrt{x}, \sqrt{z}) } \\
& \times g_{\beta-\alpha}\left(\sqrt{x},(1+z)^{1 / 2}\right),
\end{aligned}
$$

where we have used the abbreviations

$$
f_{\nu}(x, u)=(x / u)^{\nu} I_{\nu}(2 u x), \quad g_{\nu}(x, u)=(x / u)^{\nu} K_{\nu}(2 u x)
$$

for the factors that involve the modified Bessel functions. Correspondence with the Jakeman-Tough version ${ }^{19}$ is made through $x=\alpha \beta A^{2} / a^{2}$ and $z=(\beta / \alpha) \delta^{2} / a^{2}$, with $\beta=n / 2$ for an $n$-dimensional random walk in the limit of the sum $A$ of a large number of small random steps of scaled mean-squared amplitude $a^{2}$, biased by a deviation from isotropy, $\delta^{2}$, that generalizes the usual $K$ distribution. The moments of the generalized $K$ distribution are

$$
\begin{aligned}
\left\langle x^{m}\right\rangle= & {\left[\Gamma(m+\beta) / \beta^{m} \Gamma(\beta)\right]\left[\Gamma(m+\alpha) / \alpha^{m} \Gamma(\alpha)\right] } \\
& \times(\alpha \beta)^{m} F(-m, \alpha+m ; \beta ;-z),
\end{aligned}
$$


where $F(a, b ; c ; u)$ is the hypergeometric function; for $a=$ $-m$, this reduces to a polynomial in $u$ that is related to the Jacobi polynomials. By comparing moments, we find that this generalized $K$ distribution can be considered to be a multiply stochastic compounding of a simpler distribution, $Q(x, \alpha, \beta, N)$ :

$$
\begin{aligned}
K_{G}(x, \alpha, \beta, \alpha \beta+\alpha(\alpha+1) z) \\
=G(x, \beta, u) \wedge G(u, \alpha, v) \wedge Q(v, \alpha, \beta, \alpha \beta+\alpha(\alpha+1) z) \\
=K^{\prime}(x, \beta, \alpha, v) \wedge Q(v, \alpha, \beta, \alpha \beta+\alpha(\alpha+1) z),
\end{aligned}
$$

where the distribution $Q(x, \alpha, \beta, N)$ has moments

$$
\left\langle x^{m}\right\rangle=(\alpha \beta)^{m} F(-m, \alpha+m ; \beta ;-[(N-\alpha \beta) / \alpha(\alpha+1)]) \text {. }
$$

Note, however, that in this representation the $Q$ distribution is not independent of the parameters in the $K^{\prime}$ distribution.

Multiply stochastic representations for the irradiance are applicable for the model presented in Fig. 1(a), in which the integrated irradiance is detected. The operation of optical detectors is accounted for more properly by the model in Fig. 1(b), as discussed in Section 4. Nevertheless, for relatively strong irradiance fluctuations, the description given in this section may suffice.

\section{MULTIPLY STOCHASTIC REPRESENTATIONS FOR THE PHOTON- COUNTING DISTRIBUTIONS}

Discrete analogs of the distributions represented in Table 1 may be obtained by permitting these distributions to smear the mean of a Poisson distribution; i.e., they can arise as Poisson transforms of the continuous distributions. The correspondence is then to the detection model presented in Fig. $1(b)$, which is more appropriate for the operation of real photodetectors. This operation leads to the distributions presented in Table 2, in which the corresponding factorial moments are also given. Note that the factorial moments in Table 2 are identical to the direct moments of the corresponding continuous distributions in Table 1, as noted in Section 2. The exception is the noncentral negative-binomial distribution, ${ }^{34-37}$ which includes a gain factor in the Poisson distribution. Equivalent representations for the pdf's are also presented. Some well-known density functions ${ }^{27}$ are included in Table 2 for ease of comparison.

The three $K$ distributions in Table $1, K_{0}, K$, and $K^{\prime}$, transform to three discrete distributions denoted $P K_{0}, P K$, and $P K^{\prime}$. By the associative property of the smearing operation, calculation of these discrete distributions may proceed in any order desired. In particular, the $P K^{\prime}$ distribution, $A(n, \beta, \alpha$, $N$ ), is a Poisson distribution smeared by the $K^{\prime}$ distribution, but it may alternatively be viewed as a negative-binomial distribution smeared by a gamma distribution; i.e., $B(n, \beta$, $v) \wedge G(v, \alpha, N)$. This remarkable result is important for puposes of both interpretation and calculation, as is made evident in Sections 5 and 6. Properties of the negative-binomial transform are collected in Appendix A.

The three $A(n)$ distributions in Table 2 are the main subject of the remainder of this paper. They represent triply stochastic distributions and are obtained by smearing the mean of one distribution with another distribution whose mean is, in turn, smeared by a third distribution. The last one, labeled $P K^{\prime}$, encompasses the previous two, as follows:

$$
\begin{aligned}
A(n, N) & =A(n, 1,1, N), \\
A(n, \alpha, N) & =A(n, 1, \alpha, N) .
\end{aligned}
$$

Of these, $A(n, N)$ has no adjustable parameter (other than

\begin{tabular}{|c|c|c|c|c|}
\hline Name & Symbol & Expression (pdf) & $\begin{array}{l}\text { Factorial Moments } \\
\qquad(n ! /(n-m) !\rangle\end{array}$ & $\begin{array}{c}\text { Equivalent } \\
\text { Density Functions }\end{array}$ \\
\hline Poisson & $P(n, N)$ & $\left(N^{n} / n !\right) e^{-N}$ & $N^{m}$ & $\begin{array}{l}B(n, \infty, N) \\
P(n, u) \wedge \delta(u, N)\end{array}$ \\
\hline Bose-Einstein & $B(n, N)$ & $N^{n} /(N+1)^{n+1}$ & $N^{m} m !$ & $\begin{array}{l}B(n, 1, N) \\
P(n, u) \wedge E(u, N)\end{array}$ \\
\hline Negative binomial & $B(n, \alpha, N)$ & $\begin{array}{l}{\left[\alpha^{\alpha} \Gamma(n+\alpha) / n ! \Gamma(\alpha)\right]} \\
\quad \times N^{n} /(N+\alpha)^{n+\alpha}\end{array}$ & $N^{m}\left[\Gamma(m+\alpha) / \alpha^{m} \Gamma(\alpha)\right]$ & $P(n, u) \wedge G(u, \alpha, N)$ \\
\hline $\begin{array}{l}\text { Noncentral } \\
\text { negative binomial }\end{array}$ & $L(n, s, \alpha, s[u+\alpha])$ & $\begin{array}{l}{\left[s^{n} /(s+1)^{n+\alpha}\right]} \\
\quad \times \exp [-s u /(s+1)] \\
\quad \times L_{n}^{(\alpha-1)}(-u /[s+1])\end{array}$ & $m ! s^{m} L_{m}{ }^{(\alpha-1)}(-u)$ & $\begin{array}{l}B(n, k+\alpha, s[k+\alpha]) \wedge P(k, u) \\
P(n, s x) \wedge I(x, \alpha, u+\alpha)\end{array}$ \\
\hline$P K_{0}$ distribution & $A(n, N)$ & $P(n, u) \wedge E(u, v) \wedge E(v, N)$ & $N^{m}(m !)^{2}$ & $\begin{array}{l}B(n, v) \wedge E(v, N) \\
P(n, u) \wedge K(u, N)\end{array}$ \\
\hline$P K$ distribution & $A(n, \alpha, N)$ & $\begin{array}{l}P(n, u) \wedge E(u, v) \wedge \\
\quad G(v, \alpha, N)\end{array}$ & $N^{m} m !\left[\Gamma(m+\alpha) / \alpha^{m} \Gamma(\alpha)\right]$ & $\begin{array}{l}B(n, v) \wedge G(v, \alpha, N) \\
P(n, u) \wedge K(u, \alpha, N)\end{array}$ \\
\hline $\begin{array}{l}P K^{\prime} \text { distribution } \\
\text { (Whittaker) }\end{array}$ & $A(n, \beta, \alpha, N)$ & $\begin{array}{l}P(n, u) \wedge G(u, \beta, v) \wedge \\
\quad G(v, \alpha, N)\end{array}$ & $\begin{array}{l}N^{m}\left[\Gamma(m+\beta) / \beta^{m} \Gamma(\beta)\right] \\
\quad \times\left[\Gamma(m+\alpha) / \alpha^{m} \Gamma(\alpha)\right]\end{array}$ & $\begin{array}{l}B(n, \beta, v) \wedge G(v, \alpha, N) \\
P(n, u) \wedge K^{\prime}(u, \beta, \alpha, N)\end{array}$ \\
\hline$B I$ distribution & $H(n, \beta, \alpha, u+\alpha)$ & $\begin{array}{l}P(n, w) \wedge G(w, \beta, v) \wedge \\
\quad g(v, k+\alpha) \wedge P(k, u)\end{array}$ & $\begin{array}{r}{\left[\Gamma(m+\beta) / \beta^{m} \Gamma(\beta)\right]} \\
\quad \times m ! L_{m}{ }^{(\alpha-1)}(-u)\end{array}$ & $\begin{array}{l}B(n, \beta, v) \wedge I(v, \alpha, u+\alpha) \\
P(n, v) \wedge J(v, \beta, \alpha, u+\alpha)\end{array}$ \\
\hline
\end{tabular}
the mean), and $A(n, \alpha, N)$ has one adjustable parameter. The most general distribution, $A(n, \beta, \alpha, N)$, has two adjustable parameters, the primary and secondary degrees-of-freedom parameters, $\alpha$ and $\beta$, respectively. Since the last distri-

Table 2. Pdf's, Factorial Moments, and Equivalent Density Functions for Some Discrete Random Variables 
bution subsumes the others, we will study the most general one and specialize the results to discuss the simpler distributions.

The general $P K^{\prime}$ distribution $A(n, \beta, \alpha, N)$ can be represented in a bewildering variety of ways in terms of various functions of mathematical analysis, notably in terms of Whittaker functions $W_{\kappa, \mu}(x)$, as ${ }^{38}$

$$
A(n, \beta, \alpha, N)=G_{n} \exp (\alpha \beta / 2 N)(\alpha \beta / N)^{\gamma} W_{\kappa, \delta}(\alpha \beta / N),
$$

where we have used the abbreviations

$$
G_{n}=\Gamma(n+\alpha) \Gamma(n+\beta) / \Gamma(\alpha) \Gamma(\beta) n !
$$

and

$$
\gamma=(\alpha+\beta-1) / 2, \quad \kappa=-(n+\gamma), \quad \delta=(\alpha-\beta) / 2,
$$

or else in terms of confluent hypergeometric functions (or Kummer functions) $U(a, b, x)$, as $^{32}$

$$
\begin{aligned}
A(n, \beta, \alpha, N) & =G_{n}(\alpha \beta / N)^{\alpha} U(n+\alpha, 1+\alpha-\beta, \alpha \beta / N) \\
& =G_{n}(\alpha \beta / N)^{\beta} U(n+\beta, 1+\beta-\alpha, \alpha \beta / N)
\end{aligned}
$$

the last two expressions are known to be equivalent by a Kummer transformation, ${ }^{32}$ and they demonstrate the symmetry with respect to $\alpha$ and $\beta$. Special cases of Eq. (4.3) were reported previously by Bertolotti et $a .^{5}$ and by Pusey. ${ }^{6}$

We also report a generalization in which the negativebinomial distribution is smeared by a noncentral $\chi^{2}$ distribution. This distribution may suitably model the statistics of photodetection for radiation passed through turbulent media, such as the atmosphere. This is because the noncentral $\chi^{2}$ distribution provides a useful and well-known representation for laser radiation as the sum of coherent and interfering chaotic components, while the negative-binomial distribution captures the essential character of a birth-deathimmigration process, in which the death rate exceeds the birth rate. ${ }^{39}$

We have found none of the analytic representations suitable for actual calculations of the distributions. Fundamentally, the difficulties in developing suitably convergent representations for these functions stem from the essential singularities that are present both at zero and at infinite arguments. We were therefore led to develop other means of computing the distributions. We succeeded by combining two approaches; we determined a second-order recursion relation satisfied by the general distribution and then used a generalized method of steepest descent to obtain pairs of values of the probabilities for use in the recursion process. The method is discussed in Section 5 .

\section{CALCULATION OF THE PHOTON- COUNTING DISTRIBUTIONS AND FACTORIAL MOMENTS}

\section{A. Recursion Relation}

The actual evaluation of the double integral representing $A(n, \beta, \alpha, N)=P(n, u) \wedge G(u, \beta, v) \wedge G(v, \alpha, N)$ or, in more explicit form,

$$
A(n, \beta, \alpha, N)=\int_{0}^{\infty} \int_{0}^{\infty} P(n, u) G(u, \beta, v) G(v, \alpha, N) \mathrm{d} u \mathrm{~d} v
$$

can be done easily after the integration variables are changed by means of the transformation

$$
x=\alpha v / N, \quad y=\beta u / v,
$$

which corresponds to

$$
u=(N / \alpha \beta) x y, \quad v=(N / \alpha) x .
$$

For this transformation, the Jacobian $J(x, y)$ in the infinitesimal element

$$
\mathrm{d} u \mathrm{~d} v=J(x, y) \mathrm{d} x \mathrm{~d} y=|\partial(u, v) / \partial(x, y)| \mathrm{d} x \mathrm{~d} y
$$

is given by

$$
J(x, y)=\left(N^{2} / \alpha^{2} \beta\right) x,
$$

and the limits of integration remain from 0 to $\infty$ for both $x$ and $y$. If we let

$$
\lambda=N / \alpha \beta,
$$

the double integral reduces, through $u=\lambda x y, v=\beta \lambda x, J=$ $\beta \lambda^{2} x$, to

$$
\begin{aligned}
A(n, \beta, \alpha, N)= & \int_{0}^{\infty} \int_{0}^{\infty} P(n, \lambda x y) G(\lambda x y, \beta, \beta \lambda x) G(\beta \lambda x, \alpha, N) \\
& \quad \times \beta \lambda^{2} x \mathrm{~d} x \mathrm{~d} y \\
= & {\left[\lambda^{n} / n ! \Gamma(\alpha) \Gamma(\beta)\right] \int_{0}^{\infty} \int_{0}^{\infty} x^{n+\alpha-1} x^{n+\beta-1} } \\
& \times \exp [-(x+y+\lambda x y)] \mathrm{d} x \mathrm{~d} y .
\end{aligned}
$$

Therefore

$$
A(n, \beta, \alpha, N)=\left[\lambda^{n} / n ! \Gamma(\alpha) \Gamma(\beta)\right] f(n+\alpha-1, n+\beta-1, \lambda),
$$

where

$$
f(p, q, \lambda)=\int_{0}^{\infty} \int_{0}^{\infty} x^{p} y^{q} \exp [-(x+y+\lambda x y)] \mathrm{d} x \mathrm{~d} y
$$

is the double integral to be studied. We will need this function for values of $(p, q)$ that are positive but not necessarily integers.

The function $f(p, q, \lambda)$ satisfies the recurrence-differential equation

$$
\partial f(p, q, \lambda) / \partial \lambda=-f(p+1, q+1, \lambda)
$$

and, for $\lambda=0$, becomes a product of two simple integrals:

$$
f(p, q, 0)=\Gamma(p+1) \Gamma(q+1) .
$$

Repeated use of Eqs. (5.10) and (5.11) yields derivatives of all orders for $f(p, q, \lambda)$ at $\lambda=0$, but the resultant Taylor series in powers of $\lambda$ fails to converge. Instead, we derive a pair of recurrence relations for $f(p, q, \lambda)$ as follows.

We apply the divergence theorem (or Green's theorem in the plane) to the double integral that defines $f(p, q, \lambda)$ in the forms

$$
\begin{aligned}
& \iint \nabla \cdot[\hat{x} x \varphi(x, y)] \mathrm{d} x \mathrm{~d} y=\oint \hat{x} x \varphi(x, y) \cdot \mathrm{d} \mathbf{s}, \\
& \iint \nabla \cdot[\hat{y} y \varphi(x, y)] \mathrm{d} x \mathrm{~d} y=\oint \hat{y} y \varphi(x, y) \cdot \mathrm{d} \mathbf{s},
\end{aligned}
$$

where the surface integral is over the first quadrant of the $x y$ 
plane; the closed-line integral is along the boundary of that area, with ds directed along the outward normal to the bounding curve; $\hat{x}$ and $\hat{y}$ are unit vectors along the $x$ and $y$ directions; and the quantity $\varphi(x, y)$ to be used in the theorem is the integrand in Eq. (5.9),

$$
\varphi(x, y)=x^{p} y^{q} \exp [-(x+y+\lambda x y)] .
$$

Accordingly,

$$
\nabla \cdot[\hat{x} x \varphi(x, y)]=\varphi+x \partial \varphi / \partial x=(p+1-x-\lambda x y) \varphi(x, y)
$$

and

$$
\nabla \cdot[\hat{y} y \varphi(x, y)]=\varphi+y \partial \varphi / \partial y=(q+1-y-\lambda x y) \varphi(x, y) .
$$

The closed line integrals in Eqs. (5.12) and (5.13) vanish, because $\hat{x} \cdot \mathrm{d} \mathbf{s}=0$ and $\mathrm{y} \varphi=0$ along the $x$ axis and $\hat{y} \cdot \mathrm{ds}=0$ and $x \varphi=0$ along the $y$ axis; and both $x \varphi$ and $y \varphi$ vanish at an infinite distance from the origin, because of the exponential factor in $\varphi$. The result is that the divergence theorem yields

$$
\iint(p+1-x-\lambda x y) \varphi(x, y) \mathrm{d} x \mathrm{~d} y=0
$$

or

$$
(p+1) f(p, q, \lambda)-f(p+1, q, \lambda)-\lambda f(p+1, q+1, \lambda)=0
$$

and

$$
\iint(q+1-y-\lambda x y) \varphi(x, y) \mathrm{d} x \mathrm{~d} y=0
$$

or

$$
(q+1) f(p, q, \lambda)-f(p, q+1, \lambda)-\lambda f(p+1, q+1, \lambda)=0,
$$

as the two desired recurrence relations.

To use these two recurrence relations to obtain one for the probability distribution $A(n, \beta, \alpha, N)$, it is necessary to combine them, because a unit increment in $n$ causes a unit increment in both $p=n+\alpha-1$ and $q=n+\beta-1$, not just in one or the other. We need to eliminate $f(p+1, q, \lambda)$ and $f(p, q+1, \lambda)$ from the two relations, in favor of $f(p, q, \lambda)$ and $f(p \pm 1, q \pm 1, \lambda)$. We use Eq. (5.18) as it is, then Eq. (5.20) with $q$ replaced by $q-1$, and finally Eq. (5.18) again, with $(p, q)$ replaced by $(p-1, q-1)$. When $f(p+1, q, \lambda)$ and $f(p, q-1, \lambda)$ are eliminated from the three relations, the second-order recurrence relation

$$
\begin{aligned}
p q f(p-1, q-1, \lambda)= & {[1+\lambda(p+q+1)] f(p, q, \lambda) } \\
& -\lambda^{2} f(p+1, q+1, \lambda)
\end{aligned}
$$

results, in which both indices $p$ and $q$ are incremented together.

Since $A(n, \beta, \alpha, N)$ is proportional to $\left[\lambda^{n} / n !\right] f(n+\alpha-1, n$ $+\beta-1, \lambda)$, the recurrence relation [Eq. (5.21)] converts readily to one for the distribution itself:

$$
\begin{aligned}
(n+\alpha-1)(n+\beta-1) A_{n-1}= & n[2 n+\alpha+\beta-1+(\alpha \beta / N)] A_{n} \\
& -n(n+1) A_{n+1},
\end{aligned}
$$

where $A_{m}$ is an abbreviation for $A(m, \beta, \alpha, N)$ and $\lambda=N / \alpha \beta$ is used. This recurrence relation is confirmed also by combining the known recurrence properties ${ }^{32}$ of the Kummer function $U(a, b, x)$ and the gamma-function ratio $G_{n}$ in Eqs. (4.6) and (4.4).

To use this recurrence relation to compute the probability distribution, we must have two consecutive values of $A_{n}$ to start with; the relation then provides all other values in succession. It has been found that the recurrence relation is stable when used downward, to get values for smaller $n$ from those for larger $n$; that is, there is no tendency for inaccuracies in higher- $n$ values to accumulate to large errors in the values derived for lower $n$. The task remains, then, to obtain a pair of consecutive probabilities $A_{n}$ and $A_{n+1}$ for some sufficiently large $n$; these can then be used to get $A_{n-1}$ and, in turn, all the lower- $n$ values, down to $A_{0}$. What is needed is an asymptotic expression for $A_{n}$, for large $n$.

An alternative approach ${ }^{32}$ starts arbitrarily with $c A_{m+1}=$ 0 and $c A_{m}=1$ for sufficiently large $m$, with $c$ an unknown normalization constant. Use of the recurrence relation then yields $c A_{n}$ for successively smaller values of $n$, down to $n=0$, with ever-improving accuracy. The normalizing constant $c$ then can be obtained either by imposing unity as the sum of the $A_{n}$ or else by calculating any one of the $A_{n}$, say, $A_{0}$, by numerical quadrature. This procedure can yield accurate results for low values of $n$ but yields increasingly poor estimates in the tail (high-n portion) of the distribution, which is of particular interest, even when the normalizing constant has been found with high accuracy.

\section{B. Asymptotic Expression}

Accurate evaluations of the distribution $A(n, \beta, \alpha, N)$ for large $n$ can be made by adapting the method of steepest descent to the double integral that defines the distribution. We express the integrand in Eq. (5.9) as an exponential and seek its stationary point in the first quadrant of the $x y$ plane. We then expand the exponent in a Taylor series about the stationary point and retain up to quadratic terms in this series. The resultant integrand is a bivariate Gaussian with a known integral over the entire $x y$ plane. For large $n$, the errors made in neglecting higher-order terms in the Taylor series and in extending the integral to the entire plane can be made as small as desired.

We write the integral in Eq. (5.9) as

$$
\begin{aligned}
f(p, q, \lambda) & =\int_{0}^{\infty} \int_{0}^{\infty} x^{p} y^{q} \exp [-(x+y+\lambda x y)] \mathrm{d} x \mathrm{~d} y \\
& =\int_{0}^{\infty} \int_{0}^{\infty} \exp [-\psi(x, y)] \mathrm{d} x \mathrm{~d} y
\end{aligned}
$$

where

$$
\psi(x, y)=x+y+\lambda x y-p \ln x-q \ln y .
$$

The stationary point of the integrand occurs where the gradient of $\psi$ vanishes:

$$
\nabla \psi=\left(\begin{array}{l}
1+\lambda y-p / x \\
1+\lambda x-q / y
\end{array}\right)=0 .
$$

This occurs at the point $\left(x_{0}, y_{0}\right)$ such that

$$
x+\lambda x y=p, \quad y+\lambda x y=q .
$$

The Taylor series about this stationary point is 


$$
\begin{aligned}
\psi(x, y)= & \psi\left(x_{0}, y_{0}\right)+\mathbf{r} \cdot \nabla \psi\left(x_{0}, y_{0}\right) \\
& +1 / 2 \mathbf{r} \cdot \nabla \nabla \psi\left(x_{0}, y_{0}\right) \cdot \mathbf{r}+\ldots,
\end{aligned}
$$

where $\mathbf{r}=\left(x-x_{0}, y-y_{0}\right)$ and the linear term vanishes for $\left(x_{0}, y_{0}\right)$ satisfying Eqs. (5.26). The matrix of second derivatives is

$$
\nabla \nabla \psi=\left[\begin{array}{cc}
p / x^{2} & \lambda \\
\lambda & q / y^{2}
\end{array}\right],
$$

to be evaluated at $\left(x_{0}, y_{0}\right)$. The integrand then becomes, approximately,

$$
\begin{aligned}
\varphi(x, y) & =\exp [-\psi(x, y)] \approx \exp \left[-\left(\psi_{0}+1 / 2 \mathbf{r} \cdot \nabla \nabla \psi_{0} \cdot \mathbf{r}\right)\right. \\
& =\exp \left(-\psi_{0}\right) \exp \left(-1 / 2 \mathbf{r} \cdot \nabla \nabla \psi_{0} \cdot \mathbf{r}\right) \\
& =\varphi_{0} \exp \left(-1 / 2 \mathbf{r} \cdot \nabla \nabla \psi_{0} \cdot \mathbf{r}\right)
\end{aligned}
$$

and the integral itself becomes, approximately,

$$
\begin{aligned}
f(p, q, \lambda) \approx & \varphi_{0} \int_{0}^{\infty} \int_{0}^{\infty} \exp \left(-1 / 2 \mathbf{r} \cdot \nabla \nabla \psi_{0} \cdot \mathbf{r}\right) \mathrm{d} x \mathrm{~d} y \\
\approx & x_{0}^{p} y_{0}^{q} \exp \left[-\left(x_{0}+y_{0}+\lambda x_{0} y_{0}\right)\right] \\
& \times \iint_{A} \exp \left(-1 / 2 \mathbf{r} \cdot \nabla \nabla \psi_{0} \cdot \mathbf{r}\right) \mathrm{d} A .
\end{aligned}
$$

In the last integral, the domain of integration has been extended to the entire $x y$ plane, instead of only to the first quadrant, on the basis that points in the other three quadrants are so remote from $\left(x_{0}, y_{0}\right)$ that the exponent is large enough for the additional contribution to the integral to be negligible. The final integral is just the volume under the bell-shaped bivariate Gaussian surface, and its value is

$$
\begin{aligned}
\iint_{A} \exp \left(-1 / 2 \mathbf{r} \cdot \nabla \nabla \psi_{0} \cdot \mathbf{r}\right) \mathrm{d} A & =2 \pi /\left[\operatorname{det} \nabla \nabla \psi_{0}\right]^{1 / 2} \\
& =2 \pi x_{0} y_{0} /\left[p q-\left(\lambda x_{0} y_{0}\right)^{2}\right]^{1 / 2} .
\end{aligned}
$$

The approximate expression for $f(p, q, \lambda)$ is therefore

$$
\begin{aligned}
f(p, q, \lambda) \approx & 2 \pi x_{0}{ }^{p+1} y_{0}{ }^{q+1} \exp \left[-\left(x_{0}+y_{0}+\lambda x_{0} y_{0}\right)\right] \\
& \times\left[p q-\left(\lambda x_{0} y_{0}\right)^{2}\right]^{-1 / 2}
\end{aligned}
$$

provided that the stationary point $\left(x_{0}, y_{0}\right)$ is not too close to the boundary of the first quadrant of the $x y$ plane.

That point, which is where the bell-shaped surface peaks, is found by solving the pair of equations $x+\lambda x y=p$ and $y+$ $\lambda x y=q$ in Eq. (5.26). These are easily converted into a quadratic equation for the unknown $\lambda x y$ :

$$
(\lambda x y)^{2}-(p+q+1 / \lambda)(\lambda x y)+p q=0,
$$

and the solution that makes the determinant of $\nabla \nabla \psi_{0}$ positive, as is needed, is

$$
\lambda x_{0} y_{0}=(p+q+1 / \lambda) / 2-\left\{[(p+q+1 / \lambda) / 2]^{2}-p q\right\}^{1 / 2}
$$

from which we get

$$
x_{0}=p-\lambda x_{0} y_{0}, \quad y_{0}=q-\lambda x_{0} y_{0} .
$$

The approximate expression for $A(n, \beta, \alpha, N)$ is then obtained by combining relation (5.32) with Eq. (5.8) in the form

$$
\begin{aligned}
& A(n, \beta, \alpha, N) \\
& \approx \frac{x_{0}{ }^{\alpha} y_{0}{ }^{\beta}\left(\lambda x_{0} y_{0}\right)^{n} 2 \pi \exp \left[-\left(x_{0}+y_{0}+\lambda x_{0} y_{0}\right)\right]}{\Gamma(\alpha) \Gamma(\beta) n !\left[(n+\alpha-1)(n+\beta-1)-\left(\lambda x_{0} y_{0}\right)^{2}\right]^{1 / 2}},
\end{aligned}
$$

where $x_{0}, \dot{y}_{0}$, and $\lambda x_{0} y_{0}$ are determined for given $N, \alpha$, and $\beta$, and for each $n$ from Eqs. (5.34) and (5.35) with $p=n+\alpha-1$ and $q=n+\beta-1$.

The reason that this approximate, asymptotic expression can be accurate for large $n$ is that the bivariate Gaussian that was extended over the entire plane, instead of the first quadrant alone, is reduced to values less than approximately $\exp \left[-(n / \lambda)^{1 / 2}\right]$ (in the worst case of small $\alpha$ and $\beta$ ) on the $x$ and $y$ axes, compared with unity at the stationary point. The cubic and higher-order terms neglected in the Taylor series contribute even less. Hence the error in the steepestdescent approximation to the distribution can be kept as small as needed by using the approximation for sufficiently large $n$.

The procedure that we followed was to evaluate two consecutive values of the distribution for a suitably large $n$, say, $A(n+1, \beta, \alpha, N)$ and $A(n, \beta, \alpha, N)$, by the asymptotic formula (5.36) and then to apply downward recursion with the exact relation [Eq. (5.22)] to calculate $A(n-1, \beta, \alpha, N)$ and values for lower $n$ successively, down to $A(0, \beta, \alpha, N)$. This approach maintains high accuracy even for large $n$.

Asymptotic expressions for the Kummer function in Eq. (4.6), involving combinations of Bessel functions, are available as well ${ }^{32}$ and can be combined with asymptotic approximations for the gamma-function ratio in Eq. (4.4) to obtain alternative expressions for $A(n, \beta, \alpha, N)$ for large $n$.

\section{Factorial Moments}

The accuracy and the precision of the calculations were verified by calculating the apparent factorial moments of the resultant distribution, $A(n, \beta, \alpha, N)$, for comparison with the exact ones,

$$
\langle f(m, n)\rangle=N^{m} \frac{\Gamma(m+\beta) \Gamma(m+\alpha)}{\beta^{m} \Gamma(\beta) \alpha^{m} \Gamma(\alpha)} .
$$

Note that the factors such as $\Gamma(m+\alpha) / \alpha^{m}$ are just unity for $m=0$ and $m=1$ and are calculated readily for $m>1$, without recourse to the gamma function, in the form

$$
\frac{\Gamma(m+\alpha)}{\alpha^{m} \Gamma(\alpha)}=\prod_{k=1}^{m-1}(1+k / \alpha) .
$$

In practice, the factorials magnify the high-count probabilities to such an extent that only the apparent factorial moments for the lowest few values of $m$ can be calculated accurately. The variance $\left\langle n^{2}\right\rangle-\langle n\rangle^{2}$ of the distribution can be obtained by combining the factorial moments for $m=2$ and $m=1$, or directly from

$\sigma^{2}=\left\langle n^{2}\right\rangle-\langle n\rangle^{2}=N+N^{2}[(1+1 / \alpha)(1+1 / \beta)-1]$.

For $\alpha=\beta=1$, the variance reduces to $\sigma^{2}=N+3 N^{2}$, which is appropriate for the $A(n, N)$ distribution or, equivalently, the $P K_{0}, B \wedge E$, or $P \wedge E \wedge E$ distribution. For $\alpha \rightarrow \infty($ or $\beta \rightarrow \infty)$, on the other hand, the variance becomes $\sigma^{2}=N+N^{2} / \beta$ (or $N$ $+N^{2} / \alpha$ ), in accordance with the result for the negative- 
binomial distribution. Finally, for $\alpha \rightarrow \infty$ and $\beta \rightarrow \infty$, we find that $\sigma^{2}=N$, as for the Poisson distribution.

For typical choices of the mean $N$ and for $\alpha, \beta \gg 1$, the asymptotic formula applied for $n$ of the order of 100 or so gave distributions whose factorial moments agreed with the exact values to within a fraction of $1 \%$; for the worst case of $\alpha$ $=\beta=1$, it is necessary to start at a value of $n$ in excess of 400 to attain comparable accuracy. Calculations were performed on an Apple Macintosh desk-top computer, using a BASIC program running with double precision; typical cases were calculated and plotted in approximately $1 \mathrm{~min}$, by using a compiled version of the program.

\section{RESULTS FOR THE PHOTON-COUNTING DISTRIBUTIONS}

We present several examples of calculated distributions, displaying the wide variety of probability distributions encompassed by the triply stochastic $P(n, u) \wedge G(u, \beta, v) \wedge G(v$, $\alpha, N)$ construction.

Figure 2 shows the distribution $P(n, u) \wedge G(u, 3, v) \wedge G(v, 2$, 20 ), formed by driving a Poisson counting detector with light that has a gamma distribution with parameter $\alpha=2$ (possibly representing the intrinsic irradiance fluctuations of the light source) that has itself been smeared by a gamma distribution of parameter $\beta=3$ (possibly representing the fluctuations of the mean irradiance imposed by the medium). The mean count is $N=20$, and the plot is a linear one. For this case, downward recursion was begun at $n=400$, and it resulted in a calculated sum of the probabilities of 1.00579 instead of 1 , a calculated mean of 20.115 rather than 20 , and a calculated variance of 419.82 instead of the exact one, 420 . This case is fairly typical of those with $\alpha, \beta>1$. Note that, because of the symmetry property $A(n, \beta, \alpha, N)=A(n, \alpha, \beta$, $N)$, this case can equally well be considered to arise from detection of light from a source with parameter $\alpha=3$, after traversal of a medium that imposes fluctuations with parameter $\beta=2$, instead of the reverse pair of degrees of freedom.

The case of $A(n, N)=A(n, 1,1, N)=P(n, u) \wedge E(u$, v) $\wedge E(v, N)$, which is also the exponentially driven BoseEinstein distribution $B(n, v) \wedge E(v, N)$ or, equivalently, the $K_{0}$-driven Poisson distribution $P(n, u) \wedge K(u, N)$, is shown in Fig. 3, again for a mean count of $N=20$. For this extreme case, in which the degrees of freedom of the light source and of the medium are both unity, the tail of the distribution is long; the variance is large. The precision attained with $n=$

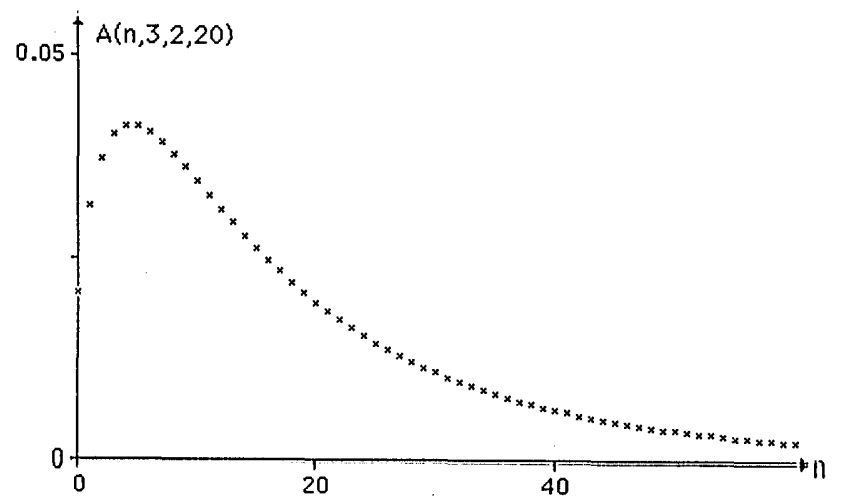

Fig. 2. Plot of the distribution $A(n, 3,2,20)=P(n, u) \wedge G(u, 3$, v) $\wedge G(v, 2,20)$ versus $n$.

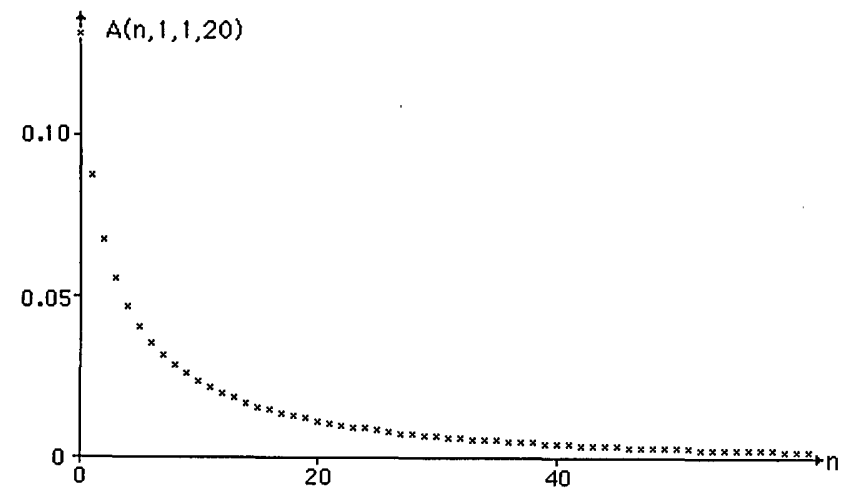

Fig. 3. Plot of the distribution $A(n, 20)=P(n, u) \wedge E(u, v) \wedge E(v, 20)$ versus $n$.

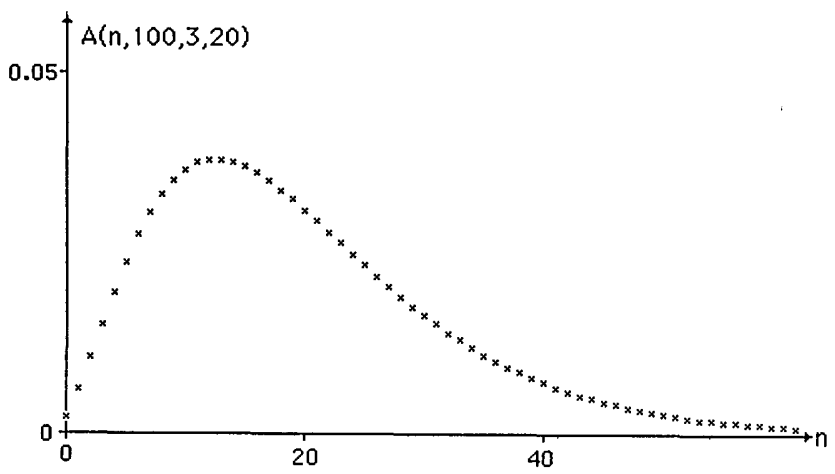

Fig. 4. Plot of $A(n, 100,3,20) \approx B(n, 3,20)$, the negative-binomial distribution, versus $n$.

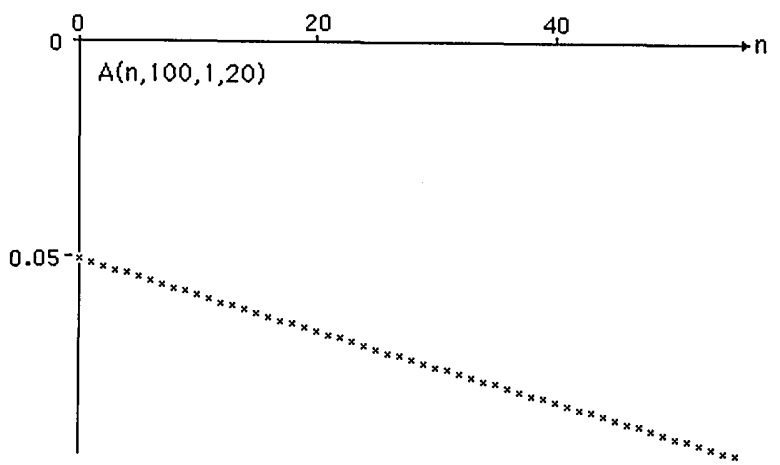

Fig. 5. Plot of $A(n, 100,1,20) \approx B(n, 20)$, the Bose-Einstein distribution, versus $n$.

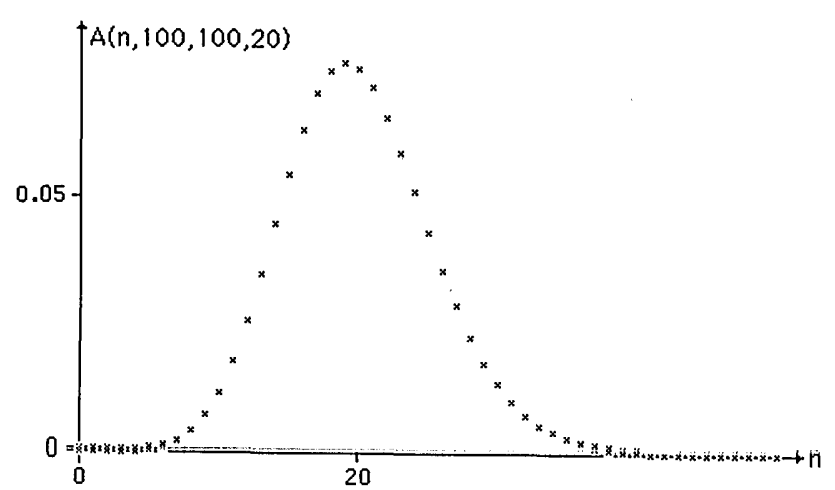

Fig. 6. Plot of $A(n, 100,100,20) \approx P(n, 20)$, the Poisson distribution, versus $n$. 
400 as the starting point for recursion is relatively poor, as evidenced particularly by the calculated variance, which was 1101.4 instead of the exact 1220; the calculated sum up to $n$ $=400$ was 1.0137 , and the apparent mean was 20.019 .

The negative-binomial distribution is obtained, approximately, for the case $A(n, 100,3,20)$, depicted in Fig. 4 . With the large value $\beta=100$ for the degrees of freedom of the medium, the $G(u, \beta, v)$ factor in the triply stochastic construction is approximately the delta-function distribution $D(u, v)$, so that $P \wedge D \wedge G$ reduces to just $P \wedge G=B(n, 3,20)$. Equivalently, the large parameter may be considered to describe the fluctuations of the light source instead. Here, the fact that $\beta$ is large aids the precision of the results: the sum up to $n=200$ is 1.00018; the mean is calculated as 20.0037; the variance is found to be 158.622 , instead of the exact 158.6667 .

Similarly, the case $\mathrm{A}(n, 100,1,20)$ approximates the BoseEinstein distribution $B(n, 20)=A(n, \infty, 1,20)$. This is just $20^{n} / 21^{(n+1)}$, which varies exponentially with $n$. Figure 5 shows a semilogarithmic plot of $A(n, 100,1,20)$ to confirm this behavior. With $n=250$ as the starting point, the calculated sum, mean, and variance are $0.99978,19.9936$, and 427.47 instead of the exact values 1,20 , and 428 , respectively.

Finally, with both $\alpha$ and $\beta$ large, the Poisson distribution is approximated fairly well by $A(n, 100,100,20)$, as shown in the linear plot in Fig. 6. The calculated sum and mean are 1.000066 and 20.0013 for $n$ up to 200; the calculated variance is 28.015 , whereas the exact one is 28.04 . The latter should be compared with 20 as the variance of the exact Poisson distribution $P(n, 20)=A(n, \infty, \infty, 20)$, showing how close $\alpha=$ $\beta=100$ is to $\alpha=\beta=\infty$ for this purpose. In this case, the large values of the degrees of freedom $\alpha$ for the source and $\beta$ for the medium can be thought of as arising from a detector integration time $T \gg \tau_{c}, \tau_{a}$. From a physical point of view, the wavelike fluctuations in both the source irradiance and its mean are washed out almost entirely, resulting in a photon-counting distribution that resembles the Poisson distribution.

\section{CONCLUSION}

The continuous $K_{0}, K$, and $K^{\prime}$ distributions are of importance for describing the field and irradiance fluctuations of scattered light, speckle, and light passed through a random medium such as the turbulent atmosphere. The $K^{\prime}$ distribution, which subsumes the others, can be understood in terms of the gamma transform of a gamma distribution, where the two distributions generally have different degrees-of-freedom parameters. The Poisson transforms of the $K_{0}, K$, and $K^{\prime}$ distributions, representing discrete photon-counting distributions, are denoted $P K_{0}, P K$, and $P K^{\prime}$, respectively. The most general of these, the $P K^{\prime}$ distribution, can be viewed as the negative-binomial transform of a gamma distribution where, again, the degrees-of-freedom parameters of the two distributions are generally different. Although all the members of this family of distributions decay slowly, they have been evaluated numerically to excellent accuracy by use of a recursion relation and a generalization of the method of steepest descent. Although these distributions can also be expressed in terms of confluent hypergeometric functions or Whittaker functions, those representations are less suitable for computational purposes.
We have also presented a convenient asymptotic form for the tail of these distributions.

We have suggested a new continuous distribution, the gamma transform of a noncentral chi-squared distribution, as a possibly useful addition to the $K$ family. We call this the $G I$ distribution, the $G$ and the $I$ representing the gamma and the modified Bessel function of the first kind in the noncentral chi-squared distribution, respectively. This distribution differs from the $H-K$, the $I-K$, and the $K_{G}$ distributions. The associated new discrete distribution in the Poisson transform domain, denoted $B I$, is the negative-binomial transform of the noncentral chi-squared distribution. The $G I$ and $B I$ distributions offer promise in modeling optical phenomena because the noncentral chi-squared distribution provides a good representation of the irradiance fluctuations of laser radiation, whereas the negative binomial captures the essence of a birth-death-immigration process in certain limits.

Finally, we point out that the results derived here may well be useful in other applications, such as auditory and visual psychophysics. ${ }^{40}$

\section{APPENDIX A: THE NEGATIVE-BINOMIAL TRANSFORM}

We collect here, for ready reference, the salient properties of counting distributions derived from an arbitrary continuous pdf by transforming it with a negative-binomial distribution; that is, the negative-binomial transform of any $\operatorname{pdf} \Phi(x$, $N$ ) is defined by

$$
\begin{aligned}
B\{\Phi\} & =B(n, \alpha, x) \wedge \Phi(x, N)=\varphi(n, \alpha, N) \\
& =\int_{0}^{\infty} B(n, \alpha, x) \Phi(x, N) \mathrm{d} x .
\end{aligned}
$$

Explicitly, this is

$$
B\{\Phi\}=\left[\alpha^{\alpha} \Gamma(n+\alpha) / n ! \Gamma(\alpha)\right] \int_{0}^{\infty}\left\{x^{n} /(x+\alpha)^{n+\alpha}\right\} \Phi(x, N) \mathrm{d} x .
$$

This operation transforms the pdf of any continuous source distribution into a discrete counting distribution. The transform is thus a three-level operation:

$$
\begin{aligned}
B\{\Phi\} & =\varphi(n, \alpha, N)=B(n, \alpha, x) \wedge \Phi(x, N) \\
& =P(n, u) \wedge G(u, \alpha, x) \wedge \Phi(x, N) .
\end{aligned}
$$

The negative-binomial kernel is of importance because the distribution arises in many contexts. ${ }^{41,42}$

The factorial moments $\langle n ! /(n-m) !\rangle$ of a negative-binomial transform $\varphi(n, \alpha, N)$ are related to the direct moments $\left\langle x^{m}\right\rangle$ of the untransformed distribution $\Phi(x, N)$ by

$$
\langle n ! /(n-m) !\rangle=\left[\Gamma(m+\alpha) / \alpha^{m} \Gamma(\alpha)\right]\left\langle x^{m}\right\rangle .
$$

The first few of these are as follows:

$$
\begin{aligned}
& \text { for } m=1,\langle n\rangle=\langle x\rangle ; \\
& \text { for } m=2,\langle n(n-1)\rangle=(1+1 / \alpha)\left\langle x^{2}\right\rangle ; \\
& \text { for } m=3,\langle n(n-1)(n-2)\rangle \\
&=(1+1 / \alpha)(1+2 / \alpha)\left\langle x^{3}\right\rangle ; \\
& \text { for } m=4,\langle n(n-1)(n-2)(n-3)\rangle \\
&=(1+1 / \alpha)(1+2 / \alpha)(1+3 / \alpha)\left\langle x^{4}\right\rangle .
\end{aligned}
$$


The probability-generating function for the transformed distribution is a function of the variable $Z$ and is defined by $G(Z)=\left\langle Z^{n}\right\rangle$. When expanded in a power series about $Z=0$, this function of $Z$ exhibits the successive counting probabilities $\varphi(n, \alpha, N)$, since

$$
G(Z)=\left\langle Z^{n}\right\rangle=\sum Z^{n} \varphi(n, \alpha, N)
$$

so that

$$
\varphi(n, \alpha, N)=(1 / n !) \mathrm{d}^{n} G(0) / \mathrm{d} Z^{n} .
$$

On the other hand, when expanded in a power series about $Z$ $=1$, the same function of $Z$ exhibits the successive factorial moments, since

$$
\begin{aligned}
G(Z)=\left\langle Z^{n}\right\rangle & =\sum[1+(Z-1)]^{n} \varphi(n, \alpha, N) \\
& =\sum \sum\left[(Z-1)^{m} / m !\right][n ! /(n-m) !] \varphi(n, \alpha, N),
\end{aligned}
$$

so that

$$
\langle n ! /(n-m) !\rangle=\mathrm{d}^{m} G(1) / \mathrm{d} Z^{m} .
$$

For the negative-binomial transform $\varphi(n, \alpha, N)$ of $\Phi(x, N)$, the probability-generating function is given in terms of an average over the original, continuous distribution $\Phi(x, N)$ as

$$
G(Z)=\left\langle Z^{n}\right\rangle=\left\langle[1-(Z-1)(x / \alpha)]^{-\alpha}\right\rangle .
$$

Examples of negative-binomial transforms of some important distributions are presented in Table 2.

\section{ACKNOWLEDGMENTS}

We are grateful to Larry Andrews, Richard A. Campos, and William J. McGill for valuable discussions. This research was supported in part by the National Science Foundation through the Center for Telecommunications Research and by the Joint Services Electronics Program through the Columbia Radiation Laboratory.

\section{REFERENCES}

1. S. R. Broadbent and D. G. Kendall, "The random walk of Trichostrongylus retortaeformis," Biometrics 9, 460-466 (1953).

2. E. J. Williams, "The distribution of larvae of randomly moving insects," Austral. J. Biol. Sci. 14, 598-604 (1961). Williams's calculation is virtually identical to that of Broadbent and Kendall. ${ }^{1}$

3. N. Yasuda, "The random walk model of human migration," Theor. Popul. Biol. 7, 156-167 (1975).

4. G. Malécot, "Identical loci and relationship," in Proceedings of the Fifth Berkeley Symposium on Mathematical Statistics and Probability; Biology and Problems of Health, L. LeCam and J. Neyman, eds. (U. California Press, Berkeley, Calif., 1967), Vol. 4, pp. 317-332.

5. M. Bertolotti, B. Crosignani, and P. DiPorto, "On the statistics of Gaussian light scattered by a Gaussian medium," J. Phys. A 3, L37-L38 (1970).

6. P. N. Pusey, "Statistical properties of scattered radiation," in Photon Correlation Spectroscopy and Velocimetry, H. Z. Cummins and E. R. Pike, eds. (Plenum, New York, 1976), pp. 45-141.
7. G. Parry, P. N. Pusey, E. Jakeman, and J. G. McWhirter, "Focussing by a random phase screen," Opt. Commun. 22, 195-201 (1977).

8. E. Jakeman and P. N. Pusey, "Significance of $K$ distributions in scattering experiments," Phys. Rev. Lett. 40, 546-550 (1978).

9. G. Parry and P. N. Pusey, " $K$ distributions in atmospheric propagation of laser light," J. Opt. Soc. Am. 69, 796-798 (1979).

10. E. Jakeman, "On the statistics of $K$-distributed noise," J. Phys. A 13, 31-48 (1980).

11. E. Jakeman, "Speckle statistics with a small number of scatterers," in Applications of Speckle Phenomena, W. H. Carter, ed., Proc. Soc. Photo-Opt. Instrum. Eng. 243, 9-19 (1980).

12. K. A. O'Donnell, "Speckle statistics of doubly scattered light," J. Opt. Soc. Am. 72, 1459-1463 (1982).

13. E. Jakeman and P. N. Pusey, "A model for non-Rayleigh sea echo," IEEE Trans. Antennas Propag. AP-24, 806-814 (1976).

14. D. J. Lewinski, "Nonstationary probabilistic target and clutter scattering models," IEEE Trans. Antennas Propag. AP-31, 490-498 (1983).

15. S. Watts and K. D. Ward, "Spatial correlation in $K$-distributed sea clutter," Proc. Inst. Electr. Eng. Part F 134, 526-532 (1987).

16. L. C. Andrews and R. L. Phillips, " $I-K$ distribution as a universal propagation model of laser beams in atmospheric turbulence," J. Opt. Soc. Am. A 2, 160-163 (1985).

17. L. C. Andrews and R. L. Phillips, "Mathematical genesis of the $I-K$ distribution for random optical fields," J. Opt. Soc. Am. A 3, 1912-1919 (1986).

18. R. Barakat, "Weak-scatterer generalization of the $K$-density function with application to laser scattering in atmospheric turbulence," J. Opt. Soc. Am. A 3, 401-409 (1986).

19. E. Jakeman and R. J. A. Tough, "Generalized $K$ distribution: a statistical model for weak scattering," J. Opt. Soc. Am. A 4, 1764-1772 (1987).

20. E. B. Rockower, "Quantum derivation of $K$-distributed noise for finite $\langle N\rangle$," J. Opt. Soc. Am. A 5, 730-734 (1988).

21. G. Parry, "Measurement of atmospheric turbulence induced intensity fluctuations in a laser beam," Opt. Acta 28, 715-728 (1981).

22. A. Consortini and R. J. Hill, "Reduction of the moments of intensity fluctuations caused by amplifier saturation for both the $K$ and the log-normally modulated exponential probability densities," Opt. Lett. 12, 304-306 (1987).

23. K. D. Ward, "Compound representation of high resolution sea clutter," Electron. Lett. 17, 561-563 (1981).

24. R. J. A. Tough, "Fokker-Planck description of $K$-distributed noise," J. Phys. A 20, 551-567 (1987).

25. C. J. Oliver and R. J. A. Tough, "On the simulation of correlated $K$-distributed random clutter," Opt. Acta 33, 223-250 (1986).

26. E. Conte and M. Longo, "Characterisation of radar clutter as a spherically invariant random process," Proc. Inst. Electr. Eng. Part F 134, 191-197 (1987).

27. B. E. A. Saleh, Photoelectron Statistics (Springer-Verlag, Berlin, 1978).

28. E. Jakeman and P. N. Pusey; "Photon-counting statistics of optical scintillation," in Inverse Scattering Problems in Optics, H.P. Baltes, ed., Vol. 20 of Topics in Current Physics (SpringerVerlag, Berlin, 1980), pp. 75-116.

29. Y. M. Lure, M. Gao, and C. C. Yang, "Probability distribution for the photocount associated with a $K$ distribution for laser intensity," J. Opt. Soc. Am. A 4(13), P84 (1987).

30. M. Greenwood and G. U. Yule, "An inquiry into the nature of frequency distributions representative of multiple happenings with particular reference to the occurrence of multiple attacks of disease or of repeated accidents," J. R. Stat. Soc. A 83, 255279 (1920).

31. J. Gurland, "Some interrelations among compound and generalized distributions," Biometrika 44, 265-268 (1957).

32. M. Abramowitz and I. A. Stegun, eds., Handbook of Mathematical Functions, Vol. 55 of National Bureau of Standards Applied Mathematics Series (U.S. Government Printing Office, Washington, D.C., 1964).

33. R. G. Laha, "On some properties of the Bessel function distributions," Bull. Calcutta Math. Soc. 46, 59-72 (1954).

34. J. Perina, "Superposition of coherent and incoherent fields," Phys. Lett. A 24, 333-334 (1967); "Superposition of thermal and 
coherent fields," Acta Univ. Palack. Olomuc. Fac. Rerum Nat. 27, 227-234 (1968); G. Lachs, "Quantum statistics of multiplemode, superposed coherent and chaotic radiation," J. Appl. Phys. 38, 3439-3448 (1967).

35. W. J. McGill, "Neural counting mechanisms and energy detection in audition," J. Math. Psychol. 4, 351-376 (1967).

36. M. C. Teich and W. J. McGill, "Neural counting and photon counting in the presence of dead time," Phys. Rev. Lett. 36, 754758 (1976).

37. S. H. Ong and P. A. Lee, "The non-central negative binomial distribution," Biom. J. 21, 611-627 (1979).

38. E. T. Whittaker and G. N. Watson, A Course of Modern Analysis, 4th ed. (Cambridge U. Press, Cambridge, 1962).
39. M. S. Bartlett, An Introduction to Stochastic Processes, $3 \mathrm{rd}$ ed. (Cambridge U. Press, Cambridge, 1978).

40. W. J. McGill and M. C. Teich, "Signal discrimination in an amplifying auditory transmission system," in Quantitative Analyses of Behavior, Vol. 10 of Signal Detection, M. L. Commons and J. A. Nevins, eds. (Erlbaum, Hillsdale, N.J., to be published).

41. J. Gurland, "A generalized class of contagious distributions," Biometrics 14, 229-249 (1958).

42. M. T. Boswell and G. P. Patil, "Chance mechanisms generating the negative binomial distributions," in Random Counts in Models and Structures, Vol. 1 of Random Counts in Scientific Work, G. P. Patil, ed., Penn State Statistics Series (Pennsylvania State U. Press, University Park, Pa., 1970), pp. 3-27. 